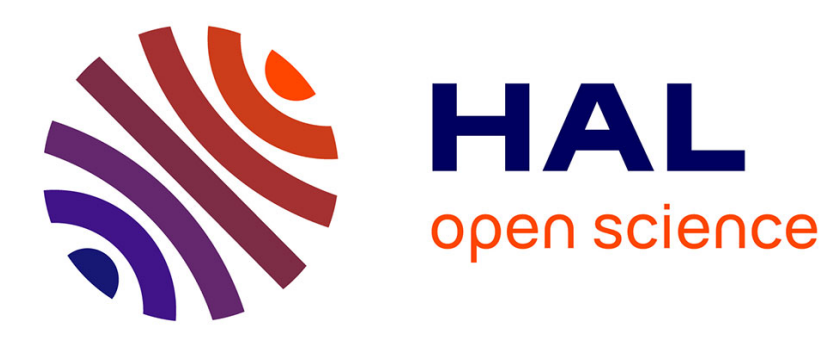

\title{
Mantle Convection Modeling with Viscoelastic/Brittle Lithosphere: Numerical Methodology and Plate Tectonic Modeling
}

Louis Moresi, Frédéric Dufour, Hans-Bernd Mühlhaus

\section{- To cite this version:}

Louis Moresi, Frédéric Dufour, Hans-Bernd Mühlhaus. Mantle Convection Modeling with Viscoelastic/Brittle Lithosphere: Numerical Methodology and Plate Tectonic Modeling. Pure and Applied Geophysics, 2002, 159 (10), pp.2335-2356. 10.1007/s00024-002-8738-3 . hal-01620839

\section{HAL Id: hal-01620839 \\ https://hal.science/hal-01620839}

Submitted on 21 Oct 2017

HAL is a multi-disciplinary open access archive for the deposit and dissemination of scientific research documents, whether they are published or not. The documents may come from teaching and research institutions in France or abroad, or from public or private research centers.
L'archive ouverte pluridisciplinaire HAL, est destinée au dépôt et à la diffusion de documents scientifiques de niveau recherche, publiés ou non, émanant des établissements d'enseignement et de recherche français ou étrangers, des laboratoires publics ou privés. 


\title{
Mantle Convection Modeling with Viscoelastic/Brittle Lithosphere: Numerical Methodology and Plate Tectonic Modeling
}

\author{
Louis Moresi,${ }^{1 *}$ Frédéric Dufour,${ }^{1}$ and Hans-Bernd Mühlhaus ${ }^{1}$
}

\begin{abstract}
The earth's tectonic plates are strong, viscoelastic shells which make up the outermost part of a thermally convecting, predominantly viscous layer. Brittle failure of the lithosphere occurs when stresses are high. In order to build a realistic simulation of the planet's evolution, the complete viscoelastic/brittle convection system needs to be considered. A particle-in-cell finite element method is demonstrated which can simulate very large deformation viscoelasticity with a strain-dependent yield stress. This is applied to a plate-deformation problem. Numerical accuracy is demonstrated relative to analytic benchmarks, and the characteristics of the method are discussed.
\end{abstract}

Key words: Mantle convection, viscoelasticity, brittle failure, finite element, Lagrangian, geodynamics.

\section{Introduction}

Solid state convection in the earth's mantle drives the surface motion of a cool thermal boundary layer comprising a number of distinct lithospheric plates. Motions in the mantle are described by the equations of fluid dynamics for substantial deformation. The rheology needed to describe deformation in the lithosphere is highly nonlinear, and near the surface where temperatures are less than approximately $600^{\circ} \mathrm{C}$ it becomes necessary to consider the role of elasticity (WATTS et al., 1980). The strong correlation between seismicity and plate boundaries (e.g., BARAZANGI and DoRman, 1969) makes it seem likely that plate motions are associated with localization of deformation occurring when stresses reach the yield strength of the lithosphere.

This picture of the earth's interior is widely accepted by geophysicists (Fig. 1). It clearly indicates that the fundamental process is thermal convection; plate tectonics is the manner in which the system organizes. Therefore, a consistent model of plate

\footnotetext{
${ }^{1}$ CSIRO Exploration and Mining, PO Box 437, Nedlands, WA 6009, Australia. E-mail: 1.moresi@ned.dem.csiro.au,http://www.ned.dem.csiro.au/research/solidmech/ F. Dufour, E-mail: frederic@ned.dem.csiro.au; H-B. Mühlhaus, E-mail: hans@ned.dem.csiro.au ${ }^{*}$ Present Address: School of Mathematical Sciences, Monash University, PO Box 28M Clayton, Victoria, 3800 Australia
} 


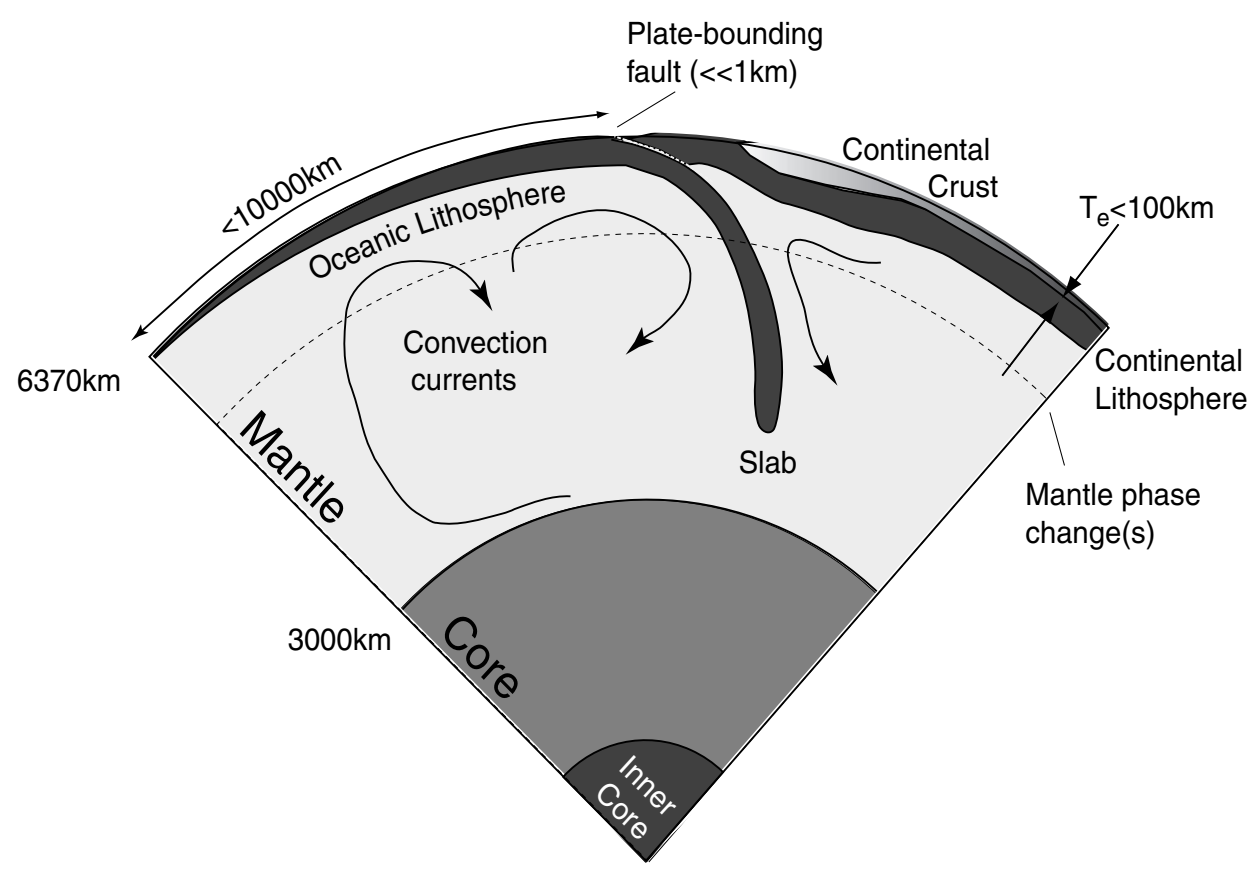

Figure 1

A simplified cross section of the earth with major layerings shown to scale except for the upper boundary layer which is exaggerated in thickness by a factor of roughly two.

behavior should contain a description of the convection system of which the plate is a part. The principle difficulty for modeling is that plate tectonics is itself only a kinematic description of the observations: a fully consistent dynamic description of the motion of the plates is still sought. Another issue is the very large range of timeand length-scales which must be considered in a complete model. For example, plates span up to $10,000 \mathrm{~km}$ horizontally, the elastic thickness is less than $100 \mathrm{~km}$, and the relevant scale of plate bounding faults may be a few hundreds of metres.

There have been major steps towards the simulation of plate tectonics in recent years by solving brittle/viscous fluid flow equations (e.g., TACKLEY, 1998, 2000; Moresi and Solomatov, 1998; Ogawa, 2001). The importance of elasticity has not been quantified by such modeling. In the past, strongly viscoelastic convection simulations with a lithosphere component have been limited to models with explicit layering in which a nonconvecting viscoelastic layer is coupled to a viscous convecting domain (PoDLADCHIKov et al., 1993). Viscoelastic mantle convection simulations have been limited to considering constant viscosity (HARDER, 1991). Models of subduction zones which incorporate viscoelasticity, faulting, and freesurface behavior have generally been limited to modest evolution times, after which further deformation produces severe remeshing problems (e.g., Melosh, 1978, GURNIS et al., 1996). 
Having identified the need for efficient, large-scale convection simulations with elastic effects in an evolving cool lithosphere, we present a method for simulating viscoelastic-brittle materials in extreme deformation. The method is first tested on a number of very simple benchmark cases in which analytic solutions are known, or where the accuracy can otherwise be quantified in large deformation. We then demonstrate the application of the method to mantle convection with a viscoelastic, brittle lithosphere.

\section{Mathematical Model}

We begin our analysis in a general way with the classical momentum conservation equation:

$$
\nabla \cdot \boldsymbol{\sigma}=\mathbf{f}
$$

where $\boldsymbol{\sigma}$ is the stress tensor and $\mathbf{f}$ a force term. As we are interested only in very slow deformations of highly viscous materials, (infinite Prandlt number) we have neglected all inertial terms in (1). It is convenient to split the stress into a deviatoric part, $\tau$, and an isotropic pressure, $p$,

$$
\boldsymbol{\sigma}=\tau-p \mathbf{I}
$$

where $\mathbf{I}$ is the identity tensor.

\section{Viscoelasticity}

We will employ a Maxwell viscoelastic model which has been used in previous studies of lithospheric deformation where viscous and elastic effects are important such as post-glacial rebound (Peltier, 1974). Schmalholz et al. (2001) provide an excellent discussion of the formulation of numerical schemes for large-deformation viscoelastic modeling of geological folding, which highlights many of the issues which our method is designed to overcome.

This model assumes that the strain rate tensor, $\mathbf{D}$, defined as:

$$
D_{i j}=\frac{1}{2}\left(\frac{\partial V_{i}}{\partial x_{j}}+\frac{\partial V_{j}}{\partial x_{i}}\right)
$$

is the sum of an elastic strain rate tensor $\mathbf{D}_{e}$ and a viscous strain rate tensor $\mathbf{D}_{v}$. The velocity vector, $\mathbf{V}$, is the fundamental unknown of our problem and all these entities are expressed in the fixed reference frame $x_{i}$. Now we decompose each strain tensor

$$
\mathbf{D}_{e}=\frac{1}{3} \operatorname{tr}\left(\mathbf{D}_{e}\right) \mathbf{I}+\hat{\mathbf{D}}_{e} \quad \text { and } \quad \mathbf{D}_{v}=\frac{1}{3} \operatorname{tr}\left(\mathbf{D}_{v}\right) \mathbf{I}+\hat{\mathbf{D}}_{v},
$$

where $\hat{\mathbf{D}}$ is the deviatoric part of $\mathbf{D}$ and $\operatorname{tr}(\mathbf{D})$ represents the trace of the tensor. 
Individually we express each deformation tensor as a function of the deviatoric stress tensor $\tau$ and pressure $p$ :

$$
\frac{\stackrel{\nabla}{\tau}}{2 \mu}+\frac{\tau}{2 \eta}=\hat{\mathbf{D}}_{e}+\hat{\mathbf{D}}_{v}=\hat{\mathbf{D}}
$$

where $\underset{\tau}{\tau}$ is the Jaumann corotational stress rate for an element of the continuum, $\mu$ is the shear modulus and $\eta$ is shear viscosity.

$$
\stackrel{\nabla}{\tau}=\frac{D \tau}{D t}+\tau \mathbf{W}-\mathbf{W} \tau
$$

where $\mathbf{W}$ is the material spin tensor,

$$
W_{i j}=\frac{1}{2}\left(\frac{\partial V_{i}}{\partial x_{j}}-\frac{\partial V_{j}}{\partial x_{i}}\right)
$$

The $\mathbf{W}$ terms account for material spin during advection which reorients the elastic stored-stress tensor.

The Jaumann derivative is an objective (observer independent) rate, although others exist which account further for the effects of a deforming coordinate system. In particular, the Olroyd derivative contains terms to account for material stretching during advection. HARDER (1991) discusses the influence of the different objective derivatives on convection solutions and finds that the stretching terms strongly effect rapidly shearing boundary layers near corners. In our proposed application where viscoelasticity is important in the stiff, cool thermal boundary layer but not elsewhere, the simpler Jaumann derivative is probably adequate. However, we plan to test this assumption in further studies.

The isotropic part provides a scalar equation for the pressure:

$$
\frac{1}{K_{e}} \frac{D p}{D t}+\frac{p}{\xi}=-\operatorname{tr}(\mathbf{D})
$$

where $K_{e}$ is the bulk modulus, $\xi$ is the bulk viscosity and $D / D t$ is a material time derivative. We note that the form of equation (8) is unsuited to conventional fluids as the material has no long-term resistance to compression. This behavior is, however, relevant to the simulation of the coupled porous-flow, matrix deformation problem where it is common to ascribe an apparent bulk viscosity to the matrix material in order to model compaction effects on large scales (e.g., McKenzIE, 1984).

\section{Brittle Failure}

Rocks in the cool lithosphere have a finite strength which may be exceeded by tectonic stresses. For a full description of the lithosphere, therefore, it is necessary to include a description of the brittle nature of the near-surface material. Here we use 
the term "brittle" quite loosely to distinguish fault-dominated deformation which may result in seismic activity, from ductile creep which occurs at higher temperature and pressure. In all recent studies of mantle convection where the brittle lithospheric rheology has been considered, the brittle behavior has been parameterized using a nonlinear effective viscosity which is introduced whenever the stress would otherwise exceed the yield value $\tau_{\text {yield }}$. This approach ignores details of individual faults, and treats only the influence of fault systems on the large-scale convective flow. It produces a lithospheric strength profile in accord with Byerlee's law (e.g., BYERLEE, 1978).

To determine the effective viscosity we extend (5) by introducing a Prandtl-Reuss flow rule for the plastic part of the stretching, $\mathbf{D}_{p}$ :

$$
\frac{\nabla}{2 \mu}+\frac{\tau}{2 \eta}+\lambda \frac{\tau}{2|\tau|}=\hat{\mathbf{D}}_{e}+\hat{\mathbf{D}}_{v}+\hat{\mathbf{D}}_{p}=\hat{\mathbf{D}},
$$

where $\lambda$ is a parameter to be determined such that the stress remains on the yield surface, and $|\tau| \equiv\left(\tau_{i j} \tau_{i j} / 2\right)^{(1 / 2)}$. The plastic flow rule introduces a nonlinearity into the constitutive law which, in general, requires iteration to determine the equilibrium state.

\section{Numerical Implementation}

As we are interested in solutions where very large deformations may occur including thermally driven fluid convection, we would like to work with a fluid-like system of equations. Hence we obtain a stress/strain-rate relation from (5) by expressing the Jaumann stress-rate in a difference form:

$$
\stackrel{\nabla}{\tau} \approx \frac{\tau^{t+\Delta t^{e}}-\tau^{t}}{\Delta t^{e}}-\mathbf{W}^{t} \tau^{t}+\tau^{t} \mathbf{W}^{t}
$$

where the superscripts $t, t+\Delta t^{e}$ indicate values at the current and future timestep, respectively. Equations (5) and (8) become respectively

$$
\tau^{t+\Delta t^{e}}=2 \frac{\eta \Delta t^{e}}{\alpha+\Delta t^{e}} \hat{\mathbf{D}}^{t+\Delta t^{e}}+\frac{\alpha}{\alpha+\Delta t^{e}} \tau^{t}+\frac{\alpha \Delta t^{e}}{\Delta t^{e}+\alpha}\left(\mathbf{W}^{t} \tau^{t}-\tau^{t} \mathbf{W}^{t}\right)
$$

and

$$
p^{t+\Delta t^{e}}=-\frac{\xi \Delta t^{e}}{\beta+\Delta t^{e}} D_{k k}^{t+\Delta t^{e}}+\frac{\beta}{\beta+\Delta t^{e}} p^{t},
$$

where $\alpha=\eta / \mu$ is the shear relaxation time and $\beta=\xi / K_{e}$ is the bulk relaxation time. We can simplify the above equations by defining an effective viscosity $\eta_{\text {eff }}$ and an effective compressibility $\xi_{\text {eff }}$ :

$$
\eta_{\mathrm{eff}}=\eta \frac{\Delta t^{e}}{\Delta t^{e}+\alpha} \quad \text { and } \quad \xi_{\mathrm{eff}}=\xi \frac{\Delta t^{e}}{\Delta t^{e}+\beta}
$$


Then the deviatoric stress is given by

$$
\tau^{t+\Delta t^{e}}=\eta_{\mathrm{eff}}\left(2 \hat{\mathbf{D}}^{t+\Delta t^{e}}+\frac{\tau^{t}}{\mu \Delta t^{e}}+\frac{\mathbf{W}^{t} \tau^{t}}{\mu}-\frac{\tau^{t} \mathbf{W}^{t}}{\mu}\right)
$$

and the pressure by

$$
p^{t+\Delta t^{e}}=\xi_{\mathrm{eff}}\left(D_{k k}^{t+\Delta t^{e}}-\frac{p^{t}}{\Delta t^{e} K_{e}}\right)
$$

To model an incompressible material $K_{e}$ and $\xi$ are made very large such that $D_{k k} \approx 0$.

Our system of equations is thus composed of a quasi-viscous part with modified material parameters and a right-hand-side term depending on values from the previous timestep. This approach minimizes the modification to the viscous flow code. Instead of using physical parameters for viscosity and bulk modulus, we use effective material properties (13) to take into account elasticity. The discretization of the stress rates in this manner produces an additional "force" term in the righthand side of (1) commonly called the internal force which consist of stresses from the previous timestep or from initial conditions (which we refer to as stored stresses).

$$
\mathbf{f}^{e, t}=\frac{\xi_{\mathrm{eff}}}{K_{e} \Delta t^{e}} \nabla p^{t-\Delta t^{e}}-\frac{\eta_{\mathrm{eff}}}{\mu \Delta t^{e}} \nabla \cdot \tau^{t-\Delta t^{e}}
$$

\section{Implementation of Yielding}

Starting from equation (9), we again express the Jaumann stress rate in difference form (in the Lagrangian particle reference frame) to give:

$$
\tau^{t+\Delta t^{e}}\left[\frac{1}{2 \mu \Delta t^{e}}+\frac{1}{2 \eta}+\frac{\lambda}{2|\tau|}+\right]=\hat{\mathbf{D}}^{t+\Delta t^{e}}+\frac{1}{2 \mu \Delta t^{e}} \tau^{t}+\frac{1}{2 \mu}\left(\mathbf{W}^{t} \tau^{t}-\tau^{t} \mathbf{W}^{t}\right) .
$$

No modification to the isotropic part of the problem is required when the von Mises yield criterion is used. At yield we use the fact that $|\tau|=\tau_{\text {yield }}$ to write

$$
\tau^{t+\Delta t^{e}}=\eta^{\prime}\left[2 \hat{\mathbf{D}}^{t+\Delta t^{e}}+\frac{1}{\mu \Delta t^{e}} \tau^{t}+\frac{1}{\mu}\left(\mathbf{W}^{t} \tau^{t}-\tau^{t} \mathbf{W}^{t}\right)\right]
$$

using an effective viscosity, $\eta^{\prime}$ given by

$$
\eta^{\prime}=\frac{\eta \tau_{\text {yield }} \mu \Delta t^{e}}{\eta \tau_{\text {yield }}+\tau_{\text {yield }} \mu \Delta t^{e}+\lambda \eta \mu \Delta t^{e}} .
$$

We determine $\lambda$ by equating the value of $\left|\tau^{t+\Delta t^{e}}\right|$ with the yield stress in (18). Alternatively, in this particular case, we can obtain $\eta^{\prime}$ directly as

$$
\eta^{\prime}=\tau_{\text {yield }} /\left|\hat{\mathbf{D}}_{\text {eff }}\right|
$$


where

$$
\hat{\mathbf{D}}_{\mathrm{eff}}=2 \hat{\mathbf{D}}^{t+\Delta t^{e}}+\frac{1}{\mu \Delta t^{e}} \tau^{t}+\frac{1}{\mu}\left(\mathbf{W}^{t} \tau^{t}-\tau^{t} \mathbf{W}^{t}\right)
$$

and $|\mathbf{D}|=\left(2 D_{i j} D_{i j}\right)^{1 / 2}$.

The value of $\lambda$ or $\eta^{\prime}$ is iterated to allow stress to redistribute from points which become unloaded. The iteration is repeated until the velocity solution is unchanged to within the error tolerance required for the solution as a whole.

\section{Computational Method}

Having devised a suitable mathematical representation of the class of problems we wish to model, we need to choose a numerical algorithm which can obtain an accurate solution for a wide range of conditions. Our formulation, while simple, still contains many difficult problems which require particular attention.

\section{Numerical Scheme}

In fluid dynamics, where strains are generally very large however not important in the constitutive relationship of the material, it is common to transform the equations to an Eulerian mesh and deal with convective terms explicitly. Problems arise whenever advection becomes strongly dominant over diffusion since an erroneous numerical diffusion dominates. In our case, the advection of material boundaries and the stress tensor are particularly susceptible to this numerical diffusion problem. Mesh-based Lagrangian formulations alleviate this difficulty, although at the expense of remeshing and the eventual development of a less-than optimal mesh configuration which increases complexity and can hinder highly efficient solution methods such as multigrid iteration.

A number of mesh-free alternatives are available: smooth particle hydrodynamics (e.g., see Monaghan, 1992 and references therein) and discrete element methods (CUNDAll and STRACK, 1979) are common examples from the fluid and solid mechanics fields, respectively. These methods are extremely good at simulating the detailed behavior of highly deforming materials with complicated geometries (e.g., free surfaces, fracture development), and highly dynamic systems. They are generally formulated to calculate explicitly interactions on a particle-particle scale, which is usually impossible for creeping flow which has no inherent timescale for stress transfer.

We have developed a hybrid approach - a particle in cell finite element method which uses a standard Eulerian finite element mesh (for a fast, implicit solution) and a Lagrangian particle framework for carrying details of interfaces, the stress history, etc. 


\section{The Particle in Cell Approach}

Our method is based closely on the standard finite element method, and is a direct development of the Material Point Method of Sulsky et al. (1995). Our particular formulation could best be described as a finite element representation of the equations of fluid dynamics with moving integration points.

A mesh is used to discretize the domain into elements, and the shape functions interpolate node points in the mesh in the standard fashion. Material points embedded in the fluid are advected using the nodal point velocity field interpolated by the shape functions. A typical updating scheme for the location, $\mathbf{x}_{p}$ of particle $p$ is

$$
\mathbf{x}_{p}^{t+\Delta t^{p}}=\mathbf{x}_{p}^{t}+\Delta t^{p} \sum_{\text {nodes }} \mathbf{v}_{n} N_{n}\left(x_{p}\right)
$$

where $\mathbf{v}$ is the nodal velocity and $N$ are the shape functions associated with the nodes, $n$, of the element in which the particle currently resides. $\Delta t^{p}$ is the timestep used in advecting the particles. In practice, a higher order scheme such as second- or fourthorder Runge-Kutta produces a more accurate result. Particle updates can be done in a predictor-corrector fashion, although to date we have found no benefit in doing this.

The problem is formulated in a weak form to devise an integral equation, and the shape function expansion produces a discrete (matrix) equation. Equation (1) in weak form, using the notation of (2) becomes

$$
\int_{\Omega} N_{(i, j)} \tau_{i j} d \Omega-\int_{\Omega} N_{, i} p d \Omega=\int_{\Omega} N_{i} f_{i} d \Omega
$$

where $\Omega$ is the problem domain, and the trial functions, $N$, are the shape functions defined by the mesh; we have assumed that no nonzero traction boundary conditions are present. For the discretized problem these integrals occur over subdomains (elements) and are calculated by summation over a finite number of sample points within each element. For example, in order to integrate a quantity, $\phi$ over the element domain $\Omega^{e}$ we replace the continuous integral by a summation

$$
\int_{\Omega^{e}} \phi d \Omega \quad \sum_{p} w_{p} \phi\left(\mathbf{x}_{p}\right)
$$

In standard finite elements the positions of the sample points, $\mathbf{x}_{p}$, andthe weighting, $w_{p}$ are optimized in advance. In our scheme the $\mathbf{x}_{p}$ 's correspond precisely to the Lagrangian points embedded in the fluid, and $w_{p}$ must be recalculated at the end of a timestep for the new configuration of particles.

Constraints on the values of $w_{p}$ originate from the need to integrate polynomials of a minimum degree related to the degree of the shape function interpolation, and 
the order of the underlying differential equation (e.g., HugHES, 1987). These Lagrangian points carry the history variables which are therefore directly available for the element integrals without the need to interpolate from nodal points to fixed integration points. In our case, the distribution of particles is usually not ideal, and a unique solution for $w_{p}$ cannot be found, or we may find we have negative weights which are not suitable for integrating physical history variables. We therefore store an initial set of $w_{p}$ 's based on a measure of local volume and adjust the weights slightly to improve the integration scheme.

There are additional complications involved in allowing integration points to move through the mesh. These include: the need to divide a particle in two when local strain becomes comparable to the size of the element in which it resides; and the need to develop an inverse mapping scheme to compute particle coordinates in the master element domain. For more details and benchmarks, see MoRESI et al. (2001).

\section{Elastic Timestep}

Note that the timestep used in advecting particles, $\Delta t^{p}$, may differ from the timestep used to calculate elastic stress rates, $\Delta t^{e}$. This is a reflection of the fact that the elastic timestep is chosen from a physical perspective whereas the advection timestep comes from the numerical representation. In general we choose $\Delta t^{p}$ to ensure that particles do not travel further than the typical dimensions of their local elements - a mesh based measure which it would be undesirable to see reflected in the elastic timestep. We may wish to impose an arbitrarily small timestep to resolve particle advection within a very fine mesh, or to account for a different physical process such as thermal diffusion or chemical reactions. To make $\Delta t^{e}$ correspondingly small would produce a very low effective viscosity, increase the role of the explicit elastic terms making up the internal forces, and potentially destabilize the solution. For problems in which elasticity does not dominate the physical response of the system, this approach is not appropriate. In order to keep the two timesteps independent, however, an averaging scheme is necessary to ensure that the stress rate is computed over the appropriate interval. In the update of internal stress we now write:

$$
\tau^{t} \leftarrow \phi\left(2 \eta_{\mathrm{eff}} \mathbf{D}^{t}+\frac{\eta_{\mathrm{eff}}}{\mu \Delta t^{e}} \tau^{t-\Delta t^{p}}\right)+(1-\phi) \tau^{t-\Delta t^{p}}
$$

where

$$
\phi=\frac{\Delta t^{p}}{\Delta t^{e}}
$$

This amounts to a running average of the stress tensor at a material point over a time $\Delta t^{e}$, and accounts correctly for rotation. 


\section{1-D Compression}

Choosing a simple, first-order differencing scheme for the stress rate poses risks if we want to obtain a robust formulation. In particular, as elasticity comes to dominate a system (e.g., examining a shorter and shorter timescale) one might expect the first-order scheme to experience difficulties. Two questions arise. What is a suitable value of $\Delta t^{e}$ to model a problem with a given relaxation time, and can we reliably approach sufficiently small values of $\Delta t^{e}$ withoutloss of stability?

A simple system which can address these questions is the compression at constant velocity of a viscoelastic (relaxation time, $\alpha=1$ ), compressibleunit square block. The velocity boundary condition, $v=0.1$, was applied until the sample had undergone $90 \%$ shortening, then it was switchedoff and the stresses were allowed to relax without further deformation. We solved

$$
\frac{1}{K_{e}} \frac{D p}{D t}+\frac{p}{\xi}=\frac{V}{h(t)},
$$

where the height of the sample, $h(t)$ is computed from the applied compression velocity, $V$. The solution for $p$ under loading $(V \neq 0)$ is

$$
p(t)=\lambda(t) \exp \left(-\frac{K_{e}}{\xi} t\right),
$$

where $\lambda$ is determined by numerical integration using

$$
\lambda=\int_{0}^{t} \frac{K_{e} V}{h(t)} \exp \left(\frac{K_{e}}{\xi} t\right) d t .
$$

During relaxation in response to past loading $(V=0)$

$$
p=p\left(t_{0}\right) \exp \left(-\frac{K_{e}}{\xi} t-t_{0}\right) \text {. }
$$

The pressure was benchmarked (Fig. 2) against the analytical solution for a given material $(\alpha=1.0)$, a given advection timestep $\left(\Delta t^{p}=0.001\right)$ and different values of the elastic observation time $\left(\Delta t^{e}\right)$. It is not surprising that taking values of $\Delta t^{e}$ comparable to or longer than the relaxation time produces inaccurate results. With $\Delta t^{e}=\alpha / 10$ or smaller, very accurate results are obtained. There is no loss of accuracy associated with the fact that we take a running average of the stress-rate in order to decouple the elastic and particle-advection timesteps. The only limitation from elasticity on the choice of particle-advection timestep is to limit the maximum value such that $\Delta t^{p} \leq \Delta t^{e}$. We also observed no difference in the accuracy of the numerical solution for $\Delta t^{e}=0.01$ for a range of $0.001 \leq \Delta t^{p}<0.01$. 


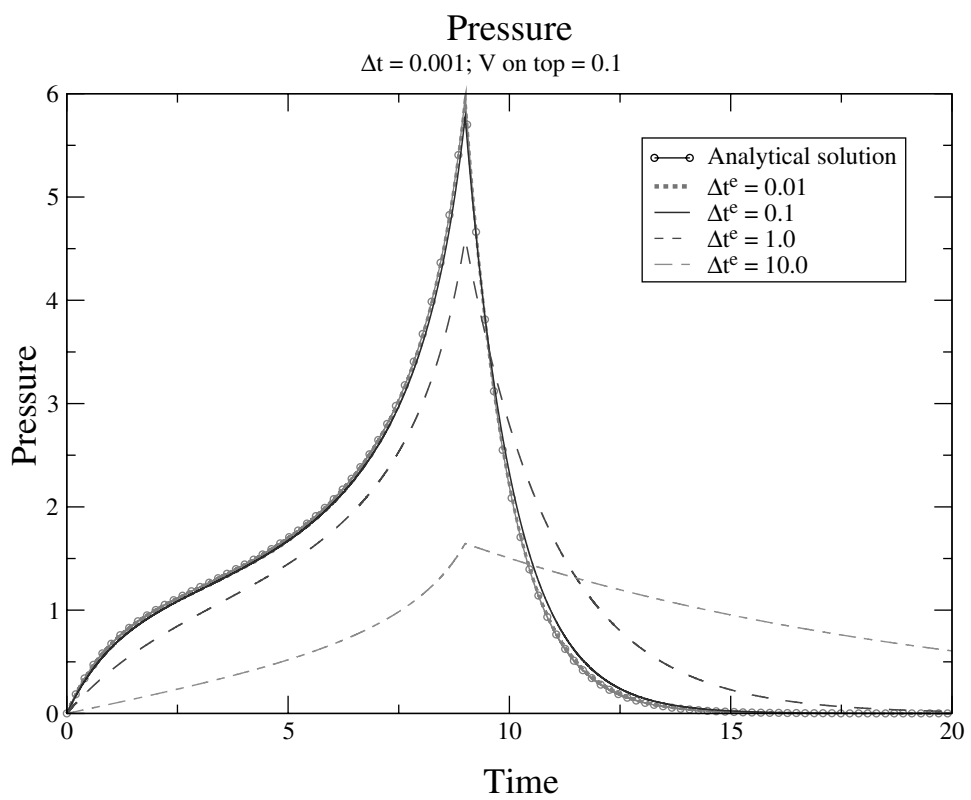

Figure 2

Numerical solution for pressure in a sample compressed in one direction for different elastic timesteps $\left(\Delta t^{e}\right)$ with fixed relaxation time $(\alpha=1)$ and particle advection timestep $\left(\Delta t^{p}=0.001\right)$. Compression starts at $t=0$ and continues until $t=9.0(90 \%$ shortening) when the sample is allowed to relax without further deformation in either direction. For comparison, the analytic solution is shown by circles.

\section{Cantilever Beam}

We next benchmark a case with material interfaces moving across mesh boundaries, and where loading and relaxation are accompanied by rotation(evaluating the importance of corotational terms in the stress transport computation). A simple example is the gravitational loading of a thick, heavy beam of viscoelastic material. The beam dimensions were 0.1 wide by 0.75 thick, with viscosity of $10^{7}$, elastic shear modulus of $10^{6}$, density 2500 and perfectly rigid support at one end. Loading was conducted in a very low viscosity $(\eta=0.01)$ compressible, background medium. The beam was loaded by applying a gravitational body force at time $t=0$, which was subsequently switched off when the deflection of the beam reached 0.35 at the unsupported end. We varied the intensity of the gravitational acceleration from $g=5$ to $g=500$ to produce responses with different characteristic times(owing to the viscous resistance of the embedding medium).

Figure 3 shows superimposed snapshots of the loading (a) and unloading (b) of the beam for $g=500$. Under such strong loading, the beam reached the maximum allowed deflection very rapidly (relative to the viscous relaxation time) and the load was then released. Once unloaded, unrelaxed elastic stress in the beam caused it to 


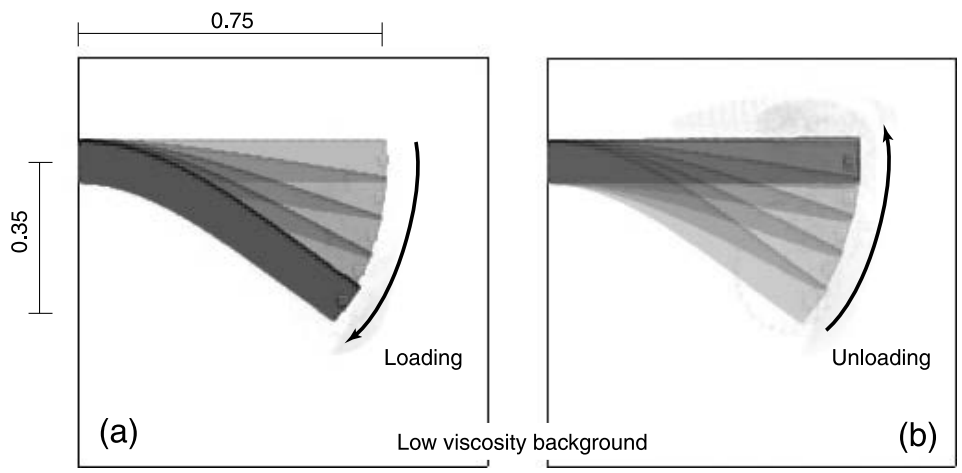

Figure 3

A heavy viscoelastic beam of length 0.75 is subjected to a gravitational load at time $t=0$ which is released when the end deflection reaches 0.35 . The superimposed configurations correspond to approximately equal time intervals.

straighten out. The loading time was sufficiently short that negligible viscous deformation occurred, allowing the beam to return to within 0.001 of its original location.

The deflection of the mid-point at the unsupported end of the beam is shown in Figure 4 for the range of loading intensities. Under light loading $(g=5,9)$ the initial deflection of the beam is rapid, dominated by elastic deformation of the beam and the viscous resistance of the embedding medium. In the absence of viscous terms, the elastic stresses in the beam would come to an equilibrium with gravitational loading. However, After $t=0.0005$, there was a small but observable rate of end deflection still occurring through gradual viscous relaxation of the elastic stresses. When the gravitational load was released, this relatively long period of viscous relaxation resulted in a permanent deflection of 0.1.In the case $g=5$, we observed the initial rapid deflection due to elastic deformation of the beam, and the gradual deflection after $t=0.0005$. However, the rate of viscous deformation was so low that the end deflection of 0.35 was not reached within the time limit imposed for the experiment.

The fast-loading cases, $g=20, g=500$, suffered very little viscous relaxation before unloading occurred. The stress state at the time of unloading was therefore similar for the two cases. Although the loading rates were very different, the unloading displacement curves remained almost parallel until most the elastic stresses had been released.

To demonstrate the importance of computing the stress update correctly, we ran the simulations omitting the corotational terms in the stress rate (dashed lines in Fig. 4). The results were qualitatively similar, however the cumulative result of not allowing for stress rotation was greater viscous deformation. This resulted in larger permanent deformation at the end of the simulation, particularly noticeable in the 


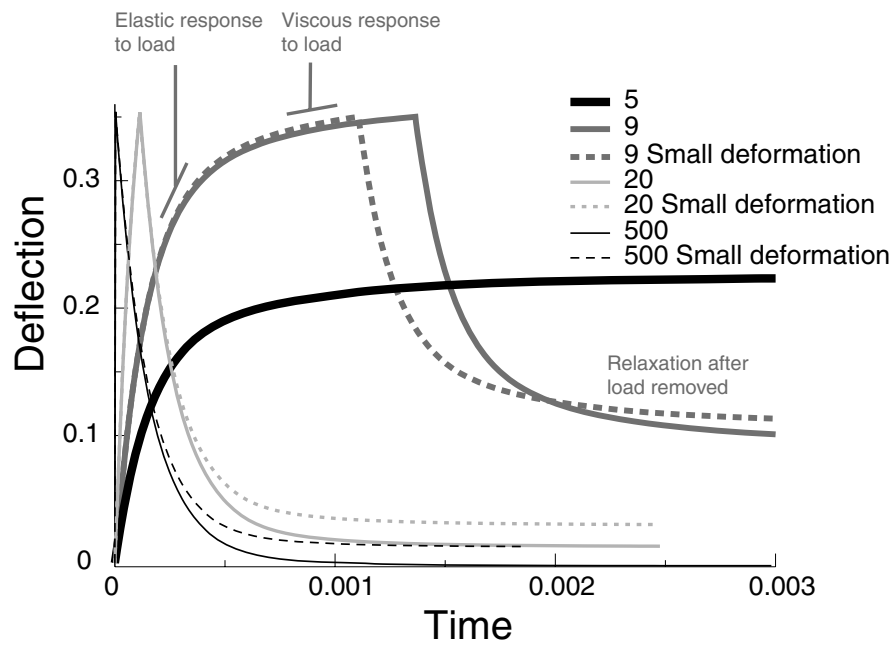

Figure 4

Deflection as a function of time for the heavy viscoelastic beam for numerous different load intensities. Dashed lines correspond to simulations where the corotational terms were omitted from the computation of the stress rate.

$g=500$ case where there is virtually no final deformation if the corotational stress rate is computed correctly.

\section{Extension of Test Sample}

The yielding algorithm is benchmarked by measuring the second invariant of the stress and displacement at points within a viscoelastic beam which was extended or compressed at a fixed rate, $v=5$, by an imposed velocity boundary condition at one end. Figure 5 indicates the geometry of the numerical experiment: the mesh was initially 3 units long by 1 unit high. The sample was 0.5 units thick, occupying the central half of the mesh, and was surrounded by a low viscosity, compressible

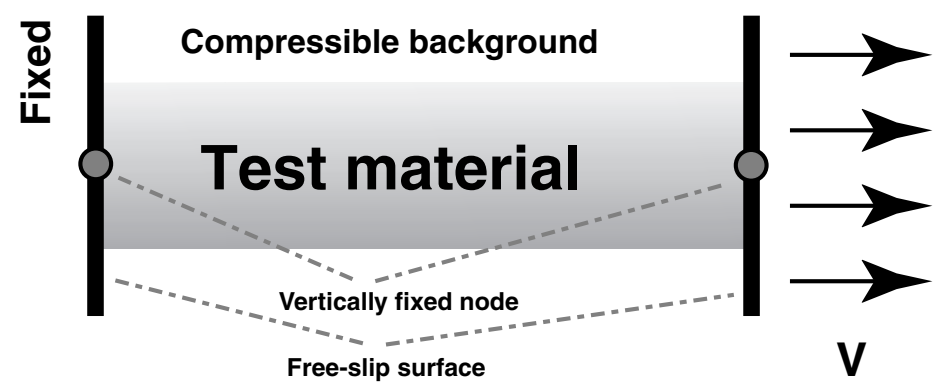

Figure 5

Geometry for simulation of the extension of a viscoelastic bar with yield stress. 


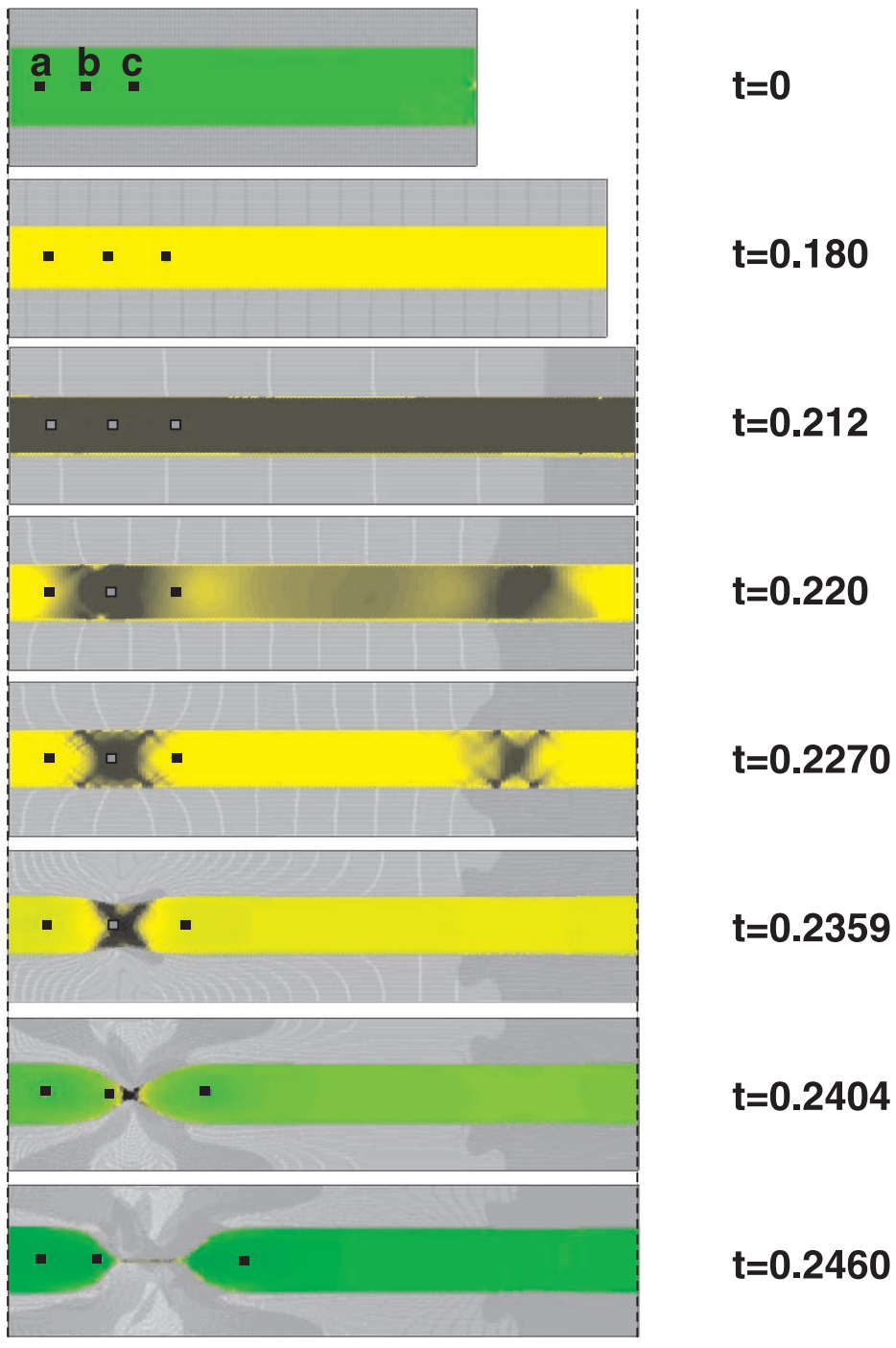

Figure 6

Simulation of the extension of a viscoelastic bar with yield stress. Black shading indicates regions deforming at yield. Embedded marker points which follow the material deformation are indicated by a,b,c.

material. Three sampling points $(a, b, c)$ for recording the stress invariant and displacement were chosen within the sample initially placed along the mid-line at $x=0.2,0.5,0.8$.

The material parameters of the sample $\left(\eta=10^{8}, \mu=10^{6}\right)$ were chosen such that the relaxation time was long $(\alpha=\eta / \mu=100)$ compared to the duration of the experiment ( 0.25$)$ so that the material behaved nearly as an elastic solid. 

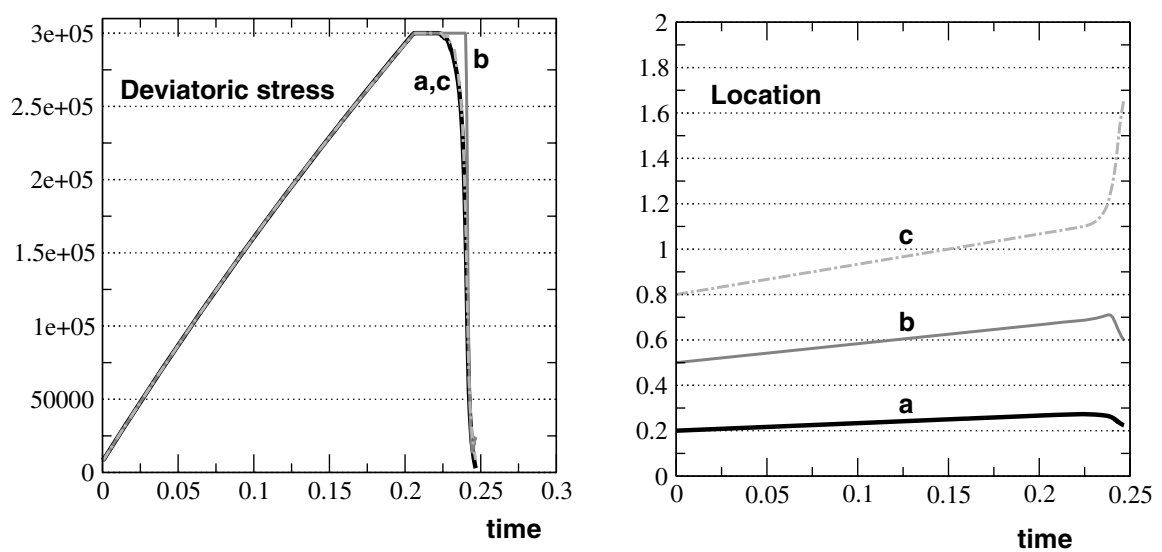

Figure 7

Stress and displacement at sample points $\mathrm{a}, \mathrm{b}, \mathrm{c}$ as a function of time for the extension experiment of Figure 6.

Figure 6 shows the progress of the experiment. Initially, deformation was uniform, resulting in gradual stretching of the sample $(t \leq 0.180)$. The entire sample reached the yield point at the same time $(t=0.212)$ and initially deformed uniformly with all points yielding. However, the deformation soon localized to a number of shear bands $(t=0.220)$, then to two places along the sample $(t=0.227)$, and finally to a single location $(t=0.2359)$ which focussed all subsequent deformation until the sample failed entirely $(t \geq 0.2404)$. The frames are not uniformly spaced in time since the post-failure behavior occurred on a considerably shorter timescale than the gradual loading. Note, for example, that the necking and separation of the two parts of the sample occurred with barely any movement of the end boundary.

Even with a material which has no strain softening, there is a tendency for deformation to localize in a particle-in-cell representation of the sample. This occurs because the sample boundary is never perfectly flat (as in real life) due to numerical fluctuations in the particle locations, and to a mild interference (Moiré) effect between the array of particles and the underlying grid. These effects produce small fluctuations in the stress field which can result in early failure at certain points. Once nucleated, shear bands can propagate from these points - ultimately resulting in necking and complete separation of the two halves of the sample.

In Figure 7a, we plot the stress at each of the sample points in the material as a function of time for a fixed end velocity. The evolution of stress within the beam was close to linear - apart from the influence of the changing of the beam thickness during deformation. The yield stress of the material was $3 \times 10^{5}$. The stress increased within the sample at the same rate for all the sample points until the yield stress was reached. At this stage, the stress was not able to increase any further, and the material deformed uniformly at the yield stress. Once localization had occurred, however, 
points outside the necking area begin to unload, and the stress dropped dramatically. The rate at which stress drops from yield back to zero is governed by the viscous part of the rheology, and the presence of a low viscosity background material.

The unloading is more clearly seen in the plots of the displacement of the sample points through time in Figure 7b. Before yielding, the displacement of each sample point increases monotonically. Once yielding occurs, and the deformation localizes, the sample points on the left of the break $(a, b)$, under the action of stored elastic stresses, rapidly retreat towards their original locations. The sample point on the right of the break (c) moves rapidly to the right as the elastic deformation relaxes.

It is worth discussing at this point a consequence of the fact that the yield criterion only applies to the deviatoric stress. During the separation of the layer, the pressure becomes enormous at the constriction, which obviously could not occur in a real material. To model this situation in a more realistic manner we would need to complement the yield criterion on the deviatoric stress with a suitable tension cutoff condition.

\section{Application to Plate Dynamics}

\section{Physical Model Description}

We treat the earth on a large scale as an incompressible, viscoelastic Maxwellfluid with infinite Prandtl number in which motions are driven by internal temperature variations. The force term from equation (1) is a gravitational body force due to density changes. We assume that these arise, for any given material, through temperature effects:

$$
\nabla \cdot \tau-\nabla p=g \rho_{0}\left(1-\alpha_{T} T\right) \hat{\mathbf{z}},
$$

where $g$ is the acceleration due to gravity, $\rho_{0}$ is material density at a reference temperature, $\alpha_{T}$ is the coefficient of thermal expansivity, and $T$ is temperature. $\hat{\mathbf{z}}$ is a unit vector in the vertical direction. We have also assumed that the variation in density only needs to be considered in the driving term (the Boussinesq approximation: BoussINESQ, 1903).

The equation of motion is then

$$
\nabla\left(\eta_{\mathrm{eff}} \mathbf{D}^{t+\Delta t^{e}}\right)-\nabla p=g \rho_{0}\left(1-\alpha_{T} T\right) \hat{\mathbf{z}}-\nabla\left(\eta_{\mathrm{eff}}\left[\frac{\tau^{t}}{\mu \Delta t^{e}}+\frac{\mathbf{W}^{t} \tau^{t}}{\mu}-\frac{\tau^{t} \mathbf{W}^{t}}{\mu}\right]\right) .
$$

The velocity field $\mathbf{u}$ and pressure at $t+\Delta t^{e}$ can be solved for a given temperature distribution and the stress history from the previous step.

Motion is driven by the heat escaping from the interior. The energy equation governs the evolution of the temperature in response to diffusion of heat through the fluid. For a given element of fluid, 


$$
\frac{D T}{D t}=-\kappa \nabla^{2} T
$$

where $\kappa$ is the thermal diffusivity of the material.

\section{Rheology}

The viscosity of the mantle at long timescale is known to be a complicated function of temperature, pressure, stress, grain-size,composition (particularly water content) etc. KARATO and WU (1993) give the following expression for mantle deformation

$$
\eta=\frac{1}{2 A}\left(\frac{\mu}{\tau}\right)^{n-1}\left(\frac{h}{b^{*}}\right)^{m} \exp \frac{E^{*}+P V^{*}}{R T},
$$

where $A$ is a constant, $\mu$ is shear modulus, $b^{*}$ is the Burgers vector, $T$ is temperature, $\tau$ is the second invariant of the deviatoric stress tensor, $E^{*}$ is an activation energy, $V^{*}$ and activation volume, $R$ is the gas constant, $h$ is the grain size, $n$ is a stress exponent, and $m$ a grain-size exponent. Despite this complexity, the dominant effect on the viscosity from the point of view of the large-scale dynamics of the system is the effect of temperature (e.g., Solomatov, 1995). To minimize the dimensionality of the parameter space we need to study, it is common to use the single-parameter Frank-Kamenetskii (McKenZIE, 1977; Moresi and Solomatov, 1995) approximation to the viscosity law to obtain the following simplification,

$$
\eta=A^{\prime} \exp (-C T)
$$

In making these assumptions, we have implicitly required that the shear modulus, $\mu$ does not vary with temperature. This is an assumption justified by seismological observations which ascribe no more than a few percent variation in seismic velocity to thermal perturbations (e.g., Woodhouse and DzIEWONSKI, 1984). In comparison to its influence on viscosity, the influence of temperature on shear modulus can be neglected. Consequently, we assume that the relaxation time of the mantle and lithosphere is entirely controlled by the viscosity. We expect to see high relaxation times only in the slowly-deforming lithosphere, and negligible relaxation times in the fast moving interior. It is this fact which makes it whatever possible to solve this problem: without relaxation of stresses in the rapidly evolving interior, we would expect unresolvably small-scale structures dominated by elastic stresses to control convection in the mantle.

\section{Mantle Convection Simulation}

The benchmarking demonstrated how complex behavior is present even in systems with the most elementary boundary conditions. We cannot hope to present a 
comprehensive examination of the influence of viscoelastic effects in the lithosphere when driven by mantle convection, and where yielding may take place at high stresses. Instead we present three convection models with different relaxation times as an indication of the styles of interaction we expect to see.

The reference simulation is for viscous convection with highly temperature dependent viscosity given by

$$
\eta=10^{5} \exp \left(-11.5129 T^{\prime}\right)
$$

where $T^{\prime}$ is dimensionless temperature varying from 0 at the surface to 1 at the base. This produces a viscosity contrast of $10^{5}$ across the entire layer. The Rayleigh number based on the interior viscosity is $3 \times 10^{6}$. The yield stress is given by

$$
\tau_{y}=10^{4}+0.4 p^{\prime},
$$

where $p^{\prime}$ is the dimensionless hydrostatic pressure. There is also a strain-softening effect: the yield stress is reduced by a factor of two linearly with strain accumulated at the yield point up to a strain of 0.5 . Above this strain, the yield stress remains constant. If a material point is heated above a dimensionless temperature of $T^{\prime}=0.5$, the accumulated strain is reset.

Simulations of this kind are capable of generating considerable realism in certain respects. For example, LeNARDIC et al. (2000), examine the interaction between subducting slabs and mobile belts using this approach with a viscous formulation.The first-order behavior of such systems is similar to that of the earth's tectonic plates: surface velocity are comparable to interior ones, large regions of the surface deform at very low strain rates compared to narrow boundary regions, and so on. However, in more detail the simulations fail to predict the dynamic behavior of subducting slabs. For example, slabs rarely roll-back (retrograde subduction) in the virtual mantle, whereas this is a near ubiquitous behaviour in the real Earth (Elsässer, 1971). Can elastic effects, which tend to unbend the slab, play a role in producing roll-back?

In the absence of elasticity the convection simulation evolved to a steady state condition with a single downwelling, a nearby surface divergence and a characteristic "rolling-forward" of the downwelling. The upper thermal boundary layer was inverted onto the lower boundary (Fig. 8a). When we introduced elastic stresses the downwelling was immediately seen to be considerably straighter (Fig. 8b) in the upper part of the mantle due to the strong elastic stresses (Fig. 8c). The evolution was also backward-rolling, i.e., with the upper thermal boundary layer landing the right way up on the lower boundary after 'subduction'.

This status was temporary, however, as the system failed to reach a thermal steady state. Strong oscillations in the surface heat transport were observed (Fig. 9a compared to 9b). The system soon evolved from rolling-back to a state with a more 
(a)

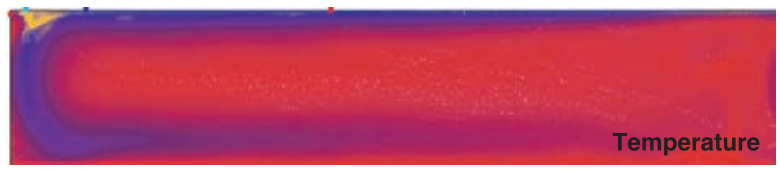

(b)

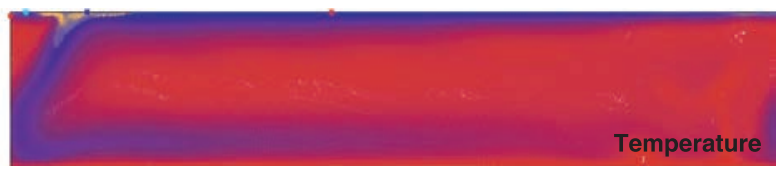

(c)

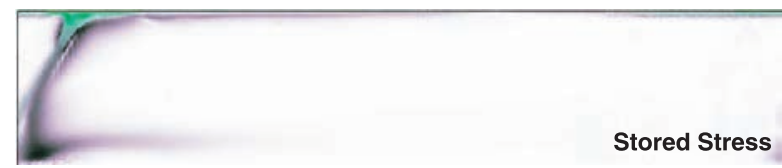

Figure 8

Example: Snapshot from two convection simulations showing $(\mathrm{a}, \mathrm{b})$ the thermal field (and regions of yielding), and (c) the magnitude of the stored stresses. The simulation in (a) has no elasticity, the simulation in $(b, c)$ has a maximum relaxation time at the surface of 0.002 compared to a surface velocity of 100 .

Regions with high strain rate due to yielding are shown in yellow.

symmetrical, stationary downwelling, and subsequently to a highly time-dependent state with rolling-forward of the downwelling.

When the relaxation time was increased from 0.002 to 0.005 , the system evolution became unexpectedly like the viscous case. The downwelling continued to roll forwards, with slight fluctuations emerging from short spells where the downwelling began to unbend. These fluctuations in the geometry of the downwelling were minor, however, with a mild influence on surface velocity, and, consequently, observed heat flow (Fig. 9c).

The influence of elasticity is to produce a pronounced tendency for the downwellings to roll backwards. In purely viscous models the tendency is to roll forwards. However, the effect of increasing the elastic stresses in the end is to modify the manner in which the lithosphere yields. This highlights the unpredictability of complex nonlinear systems and serves as a warning that application of these simulations to modeling of plate tectonics requires considerable care and a thorough attention to data which constrain the evolution of specific plate boundaries.

Obviously, this model is substantially simplified compared to the earth - the lack of a third dimension, curvature, and continents are clearly deficiencies which must be addressed. Fortunately, however, the introduction of these complicating details does not require any new algorithm development, only considerably greater computational resources. 


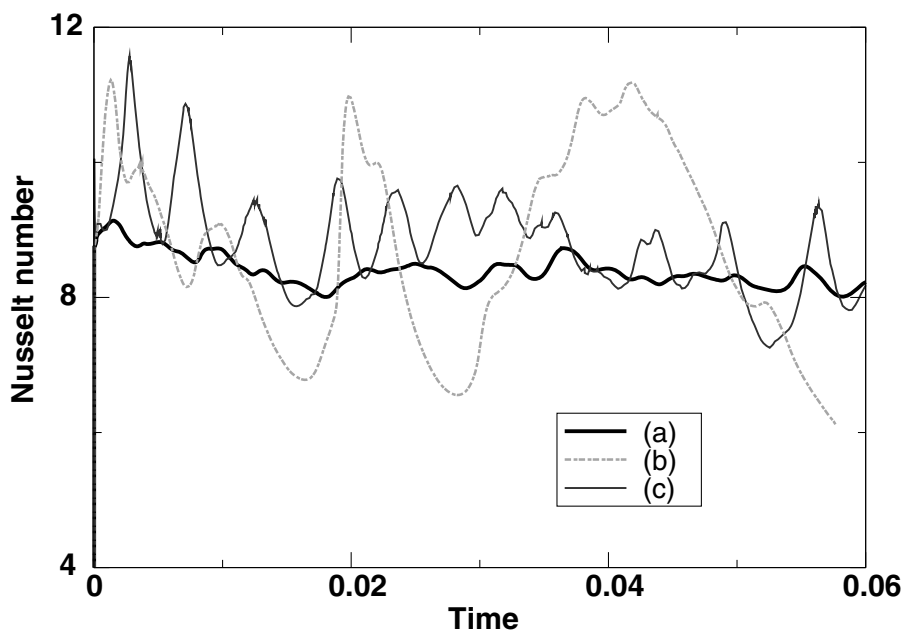

Figure 9

Plot of Nusselt number (a measure of hear transport efficiency) against time for three simulations with (a) purely viscous rheology, (b) relaxation time 0.002 , (c) relaxation time 0.005 . Relaxation times are based on the viscosity at the surface.

\section{Discussion}

We have demonstrated a model consisting of a set of rheological relations and a computational scheme for viscoelastic-plastic materials. The algorithm is designed to introduce elastic effects into convection simulations where temperature-dependent viscosity and yielding dominate the mechanical behavior. The viscosity of the mantle and the mantle lithosphere is very strongly dependent on temperature(several orders of magnitude variation over $1000^{\circ} \mathrm{C}$ ) whereas the shear modulus is not strongly affected (there is only a modest change in seismic wavespeed due to temperature). Therefore, elastic effects become unimportant outside the cold thermal boundary layer where viscosity is extremely large. The influence of elastic stresses is likely to be felt at the subduction zones where the lithosphere is bent into the interior of the earth. In these regions stresses are typically close to the yield stress - a fact which allows the plates to move in the first place.

Our methodology is limited to a coarse continuum description of the subduction zone system at a resolution of a few $\mathrm{km}$. This may give us valuable information into the nature of plate tectonics, the thermal conditions in and around subducting lithosphere, and the stress state of the system. However, the resolution is too coarse to provide any information about the detailed mechanics of the failure of lithospheric fault zones and the conditions for a major failure to occur. For this we require a coupling of the large-scale code with an engineering-scale code (e.g., DEM or smalldeformation Lagrangian FEM) using the large-scale to provide boundary conditions 
for the small scale. The issue of scale-bridging is important in many areas of numerical simulation. Essentially the same difficulties arise in material science where the atomic scale is best treated by molecular dynamics codes however the large scale must be treated as a continuum (e.g., BERNHOLC, 1999).

\section{REFERENCES}

BARAZANGi, M. and Dorman, J. (1969), World seismicity maps compiled from ESSA, Coast and Geodetic Survey, Epicenter Data 1961-1967, Bull. Seismol. Soc. Am. 59, 369-380.

Bernholc, J. (1999), Computational Materials Science: The Era of Applied Quantum Mechanics, Physics Today 52, (9), 30-35.

BoussinesQ, J. Théory analytique de la chaleur mise en harmonie avec la thermodynamique et avec la théory de la Lumière. Vol II, (Gauthier-Villars, Paris (1903)).

Byerlee, J. D. (1978), Friction of Rocks, Pure Appl. Geophys. 116, 615-629.

Cundall, P. A. and Strack, O. D. L. (1979), A Discrete Numerical Model for Granular Assemblies, Geotechnique 29 (1), 47-64.

Elsasser, W. M. (1971), Sea-floor Spreading as Thermal Convection, J. Geophys. Res. 76, 1101-1112.

Gurnis, M., Eloy, C., and Zhong, S. (1996), Free-surface formulation of mantle convection-II. Implications for subduction zone observables, Geophys. J. Int. 127, 719-727.

Harder, H. (1991) Numerical Simulation of Thermal Convection with Maxwellian Viscoelastity, J. NonNewt. Fl. Mech. 39, 67-88.

Hughes, T. J. R. The Finite Element Method, (Prentice-Hall, New Jersey, 1987)

Karato, S.-I. and Wu, P. (1993), Rheology of the Upper Mantle: A Synthesis, Science 260, 771-778.

Lenardic, A., Moresi, L., and Mühlhaus, H.-B. (2000), The Role of Mobile Belts for the Longevity of Deep Cratonic Lithosphere: The Crumple Zone Model, Geophys. Res. Lett. 27, 1235-1239.

McKenzie, D. P. (1977), Surface Deformation, Gravity Anomalies, and Convection, Geophys. J. R. Astr. Soc. 48, 211-238.

McKenzie, D. (1984), The Generation and Compaction of Partially Molten Rock, J. Petrology 25, 713-765.

Melosh, H. J. (1978), Dynamic Support of the Outer Rise, Geophys. Res. Lett. 5, 321-324.

Monaghan, J. J. (1992), Smoothed Particle Hydrodynamics, Annu. Rev. Astron. Astrophys. 30, $543-574$.

Moresi, L., Mühlhaus, H.-B., and Dufour, F., Viscoelastic Formulation for Modelling of Plate Tectonics, In Bifurcation and Localization in Soils and Rocks 99. (Mühlhaus, Dyskin, A. and Pasternak, E., ed, (Balkema, Rotterdam, 2001).

Moresi, L. and Solomatov, V. S. (1995), Numerical Investigations of 2-D Convection in a Fluid with Extremely Large Viscosity Variations, Phys. Fluids 7, 2154-2162.

Moresi, L. and Solomatov, V. S. (1998), Mantle Convection with a Brittle Lithosphere: Thoughts on the Global Tectonics Styles of the Earth and Venus, Geophys. J. Int. 133, 669-682.

Ogawa, M. (2001), The Plate-like Regime of a Numerically Modelled Thermal Convection in a Fluid with Temperature-, Pressure-, and Stress-history-dependent Viscosity, J. Geophys. Res., submitted.

Peltier, W. R. (1974), The Impluse Response of a Maxwell Earth, Rev. Geophys. Space Phys. 12, 649-669.

Podlachikov, Yu., Lenardic, A., Yuen, D. A., and Quareni, F. (1993), Dynamical Consequences of Stress Focussing for Different Rheologies: Earth and Venus Perspectives, EOS Trans. AGU, 74 (43) Suppl., 566.

Schmalholtz, S. M., Podladchikov, Y. Y., and Schmid, D. W. (2001), A Spectral/Finite Difference Method for Simulating Large Deformations of Heterogeneous, Viscoelastic Materials, Geophys. J. Int. 145, 199-208.

Solomatov, V. S. (1995), Scaling of Temperature- and Stress-dependent Viscosity Convection, Phys. Fluids, 7, 266-274.

Sulsky, D., Zhou, S.-J., and Schreyer, H. L. (1995), Application of a Particle-in-cell Method to Solid Mechanics, Comput. Phys. Commun. 87, 236-252. 
TAckley, P. J. (1998), Self-consistent Generation of Tectonic Plates in Three-dimensional Mantle Convection, Earth Planet. Sci. Lett. 157, 9-22.

TACKLEY, P. J. (2000), The quest for self-consistent generation of plate tectonics in mantle convection models, AGU Monograph on The History and Dynamics of Global Plate Motions, (ed. M. Richards), in press.

Watts, A. B., Bodine, J. H., and Ribe, N. M. (1980), Observations of Flexure and the Geological Evolution of the Pacific Basin. Nature, 283, 532-537.

Woodhouse, J. H. and Dziewonski, A. M. (1984), Three dimensional Mapping of Earth Structure by Inversion Of Seismic Waveforms, J. Geophys. Res. 89, 5953-5986. 